\title{
An emergency situation simulator for examining time-pressured decision making
}

\author{
A. LYNN FRANKLIN and EARL HUNT \\ University of Washington, Seattle, Washington
}

\begin{abstract}
Between deliberate planning efforts and reactionary decision making lies a realm of timepressured decision making. A number of real-world occupations deal with this realm on a regular basis, including air traffic controllers and emergency service dispatchers. To study this type of decision making, an emergency situation simulator has been developed, which recreates the stressful environment of the emergency service dispatcher by generating realistic hypothetical emergency situations.
\end{abstract}

Cognitive psychology has traditionally concerned itself with the investigation of mental processes that occur in two widely different time frames. Information processing psychology deals with functions such as priming or sentence comprehension, which occur within seconds or less. Very high-level problem solving and planning actions can take as little as several minutes, or as much as hours. Time-pressured decision making lies between these realms. For a decision to qualify as a time-pressured decision, four conditions must be met. First, there must be pressure to make the decision as rapidly as possible. Second, the decision itself must require the integration of multiple sources of information. If a one-dimensional judgment is all that is required, decision making reduces to the familiar choice-reaction situation. Third, there must be several alternative responses to choose from. Without a choice between policies, where is the decision making? Finally, there must be significant consequences resulting from the selection of one alternative over another. Without a reward structure, there is no pressure to make a good decision.

It is not hard to imagine situations that qualify as timepressured decisions. Most emergency decision making in human life qualifies. What is hard is to study such situations. The word emergency is defined as "a sudden, generally unexpected occurrence or set of circumstances demanding immediate action" (Webster's, 1976). Intrusive psychologists are not welcome at such times. Furthermore, field studies are plagued by problems of incomplete or lost data sets, uncontrollable factors, and a general lack of repeatability.

The laboratory provides a far more attractive experimental environment, but is it a realistic one? Laboratory

This research was supported by a subcontract between the University of Washington and Battelle Corporation, as part of a larger contract between the Battelle Corporation and the Office of Naval Research. The opinions expressed herein should not be considered opinions of the Office of Naval Research. The authors express their thanks to the personnel of the Kent Valley Public Safety Communication Center, King County, WA, for their assistance in our work. Correspondence should be addressed to E. Hunt, Department of Psychology NI-25, University of Washington, Seattle, WA 98195. studies are often criticized for having removed interesting psychological variables, in a quest for control over the experimental situation. This criticism is particularly cogent in the case of time-pressured decision making.

In our studies of time-pressured decision making, we are attempting to have the best of both worlds, by using traditional laboratory procedures to predict behavior in simulated decision-making situations, where the simulation has considerable face validity. In order to ensure the generality of our results we are considering two simulations of time-pressured decision making: air traffic control and public safety (911) dispatching. We have chosen these tasks because it is well known that they satisfy the requirements of time-pressured tasks, and because the two tasks have interesting parallels and contrasts. These points will be developed in subsequent papers. Here we concentrate on the simulated tasks themselves.

There are good commercially available simulators for air traffic control. Since they allow us to establish predefined scenarios, we have simply adopted the commercial products. However, there is no such simulator for public safety dispatching. Therefore we have developed one, which we shall now describe.

\section{THE PUBLIC SAFETY DISPATCHING TASK}

Public safety dispatching covers police, fire, and medical emergencies. We shall concentrate on police dispatching. The decision to limit our studies to police dispatching was based on differences between police dispatching and the other types of emergency dispatching, as well as the similarity of police dispatching to the tasks of air traffic controllers. Furthermore, it is common practice in dispatch centers to separate these emergency services and assign the dispatching duties to different individuals. Usually no dispatcher is required to simultaneously dispatch police and fire/medical resources.

The tasks of the police dispatcher can be divided into two major categories: (1) managing emergency situations or incidents, and (2) logging significant events into a computer record. Managing incidents involves assessing in- 
coming incident information, assigning (dispatching) responding units, and supporting assigned units by accessing and passing along supporting information. In assessing incoming information, the dispatcher makes decisions regarding the completeness and understandability of the information. The skilled dispatcher becomes a valuable source of information for the police unit. When determining how to approach a potentially dangerous situation, the police unit relies on the dispatcher's understanding and interpretation of the incident. In dispatching units, the dispatcher must consider a complex array of factors, ranging from maintaining a balance of unit availability across the city to reserving time for units to enjoy an occasional rest. In providing supporting information, the dispatcher accesses computerized driving records and personal history records and confirms the validity of outstanding warrants and restraining orders.

In addition to dispatching and supporting police units, the dispatcher must log all significant events into a computer record. Such events range from periodic status checks of the police units (to make sure they are safe and healthy) to detailing activities as they occur during the resolution of an incident. Whenever a police unit changes location or takes some action, the computer log of that incident must be updated.

The number of police units that fall under the responsibility of a single dispatcher can be as large as 20 or more. As events stack up, the dispatcher has to perform a number of very similar and often repetitive tasks for several units simultaneously. The cognitive load on working memory can vary tremendously from moment to moment. As the result of our investigations, we have developed a healthy respect for good dispatchers.

Dispatchers typically work at computerized workstations with multiple CRT monitors. Each monitor displays a variety of information, some of it redundant. In general, however, the different regions of the displays are intended to provide different types of information. In addition to controlling the displays, the dispatcher must coordinate incoming and outgoing information by using the computer keyboard, as well as maintain traditional radio and telephone communication.

\section{THE DESIGN OF THE SIMULATOR}

The DISPATCHER program has been designed to incorporate the major communication features available to professional dispatchers. The program was written in $\mathrm{C}++$ for the Macintosh, using object-oriented design techniques. It can start with 1 MB of RAM, but as the simulation continues, up to $2 \mathrm{MB}$ may be required. Although the program runs on a Macintosh, it definitely does not have the feel of a Macintosh program. This is because it was intended to simulate what a dispatcher does when using the equipment found in the center. Figure 1 provides the general layout of the screen display. This looks very much like a dispatcher display, with two exceptions. Radio and telephone communication is simulated by text and keystrokes, and all information is on a single display instead of two. Next, we shall describe the various windows in the display.

The Status Window, shown in Figure 2, is used to display information about the availability of police units, the number and type of incidents waiting for resolution, and the status of the active incidents that are currently being resolved.

The Dispatch Window, shown in Figure 3, displays detailed information about individual incidents. Dispatchers use this information, in conjunction with availability and priority information, to decide on unit assignments.

The History Window is used to display information retreived from computer records. This information can be a history of past events for a particular police unit or incident, or it may be information obtained from searches of license plates and personal records. Figure 4 provides

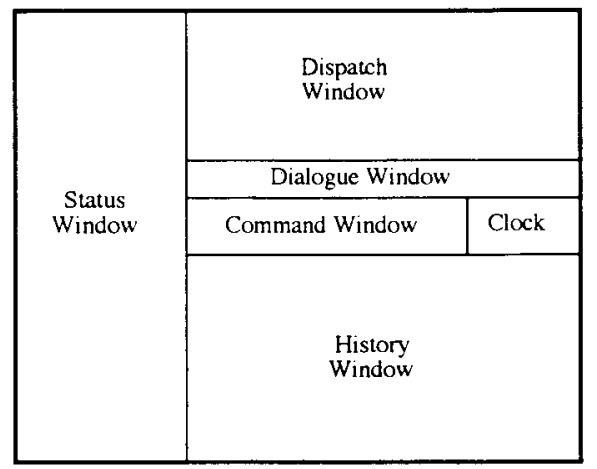

Figure 1. DISPATCHER screen layout.

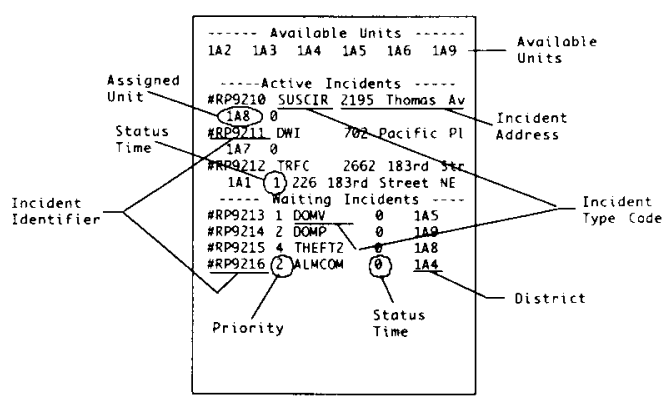

Figure 2. Typical DISPATCHER Status Window display.

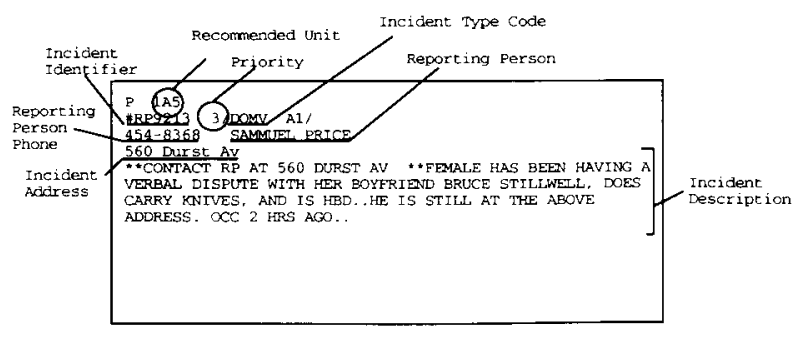

Figure 3. Typical Dispatch Window display. 


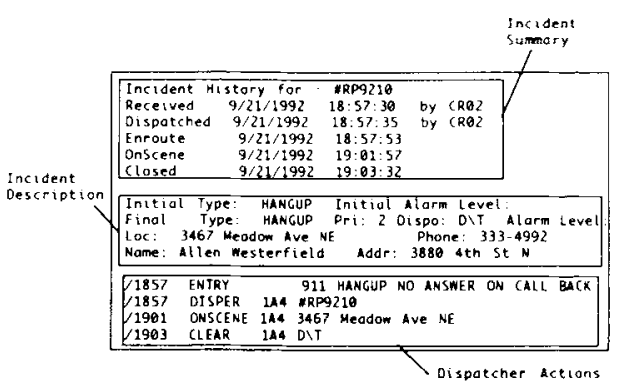

Figure 4. Typical History Window display.

a typical History Window display of past events for a specific incident.

The Clock Window, shown in Figure 5, is just a timeof-day clock. It plays only a minor role in the activities of the dispatcher, but it has been included in the display to retain simulation fidelity. The Command Window, also shown in Figure 5, displays commands as they are entered by the user. This window is used as an entry buffer, permitting the correction of typographical errors before commands are sent to the simulator.

The last window, the Dialogue Window (see Figure 5), is unique to the DISPATCHER program. Professional dispatchers receive considerable information over telephone and radio. The dialogue window was developed to simulate communication messages that would normally arrive from police units over the radio or telephone. The arrival of each message in the Dialogue Window is announced with a tone to help simulate the reception of auditory information.

Color is used to communicate information about incident priority and police unit status both in the simulation and in the field. Colors are used to provide information about the status of police units and the priorities of waiting incidents. The program will run on a black and white monitor, but, obviously, the color information will then be lost.

\section{PROGRAM STRUCTURE}

\section{Object-Oriented Design}

DISPATCHER has been built from an object-oriented design approach, with three major classes of objects existing within the application: (1) the Wizard, (2) scenarios, and (3) players. The overall flow of events within DISPATCHER is controlled by the Wizard object. In some sense, the Wizard reflects the effect of fate in the real world. From the perspective of the professional dispatcher, fate determines when new incidents occur. Within DISPATCHER, the Wizard oversees the creation of new incidents and determines which police units will be available to the dispatcher. The Wizard also controls the synchronization of the application by coordinating and processing the events of the simulator. A specific description of simulator events will be provided in more detail later in this paper. Finally, the Wizard is responsible for noting when screen updates should occur. All the procedures for writing to the screen belong to the Wizard object.

Each incident or crime that occurs within DISPATCHER is created as a separate scenario object. Scenario objects keep track of whether or not police units have been assigned to an incident and which police units have been attached to it. The scenario objects are integrally involved in the scoring process by monitoring activities that occur during the resolution of that incident. As part of this task, the scenario objects interact with the Wizard object to ensure that the assignments of police units are completed properly. Improper assignments result in penalty points, which lower the score obtained by the dispatchers (subjects). One of the more important functions of the scenario objects is to control what information is available to which characters at various times. For example, in the case of a suspicious subject or a burglary, the name of the subject should not be available to the dispatcher or the police unit until the suspect has come in contact with the police unit.

The player objects are the most complex of the three object types. To manage this complexity, the player class of objects has been further subdivided into subclasses. Currently, subclasses are defined only for police units and burglars. These subclasses were used to resolve design issues associated with object-to-object interaction. The degree of complexity that can be achieved through the interaction of these subclasses surpasses that needed to maintain situation fidelity. The police object subclass contains all of the behavioral procedures used to support the interactive session between the user (dispatcher) and the police unit. The behavioral characteristics are currently coded directly into the objects themselves. Coding the behavioral knowledge into rule sets and using production rule inferencing was briefly considered for DISPATCHER but was rejected to simplify the control structure of the program.

\section{Custom Event-Driven Activities}

In traditional programs, entering information on the keyboard temporarily suspends all other processing until the data entry activity is completed. This transfer of control to the keyboard can disrupt the simulation of emergency situations. It is much more desirable to create a control structure in which keystrokes are buffered (stored) until the data have been entered, so that the input can be

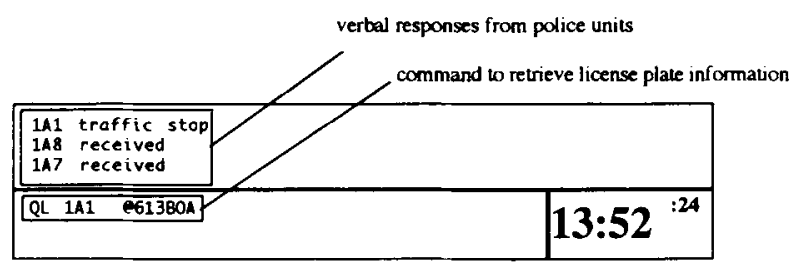

Figure 5. Typical displays for the Clock, Dialogue, and Command Windows. 
processed without delay. Therefore, DISPATCHER processes user keystrokes as background activities. This technique for keyboard processing denies the user the opportunity to artificially stall the simulation by pressing a key and then pausing to consider options while the keyboard has control of the program.

The desire to maintain simulator activity during slow data entry by the user led to the development of DISPATCHER's custom event driver. The event driver is custom in that it does not make use of the event mechanism built into the Macintosh operating system. Within the DISPATCHER, the Macintosh event loop is used solely to watch for keystrokes. When a keystroke is made, the Macintosh event manager takes control of the program for just enough time to decode the key, look for one of a small set of possible single keystroke commands, and buffer the keystroke if a single keystroke command has not been found. One of the possible single keystroke commands is the RETURN key, which directs the DISPATCHER to process the keystroke buffer. All other actions within DISPATCHER, including screen refreshing, are handled as custom events and are managed by the Wizard object.

Custom events contain information about the type of event, the time at which the event should occur, which object is to perform the action, and what that action is to be. Within DISPATCHER, the Wizard, along with each scenario and player object, can post events to the event list. The Wizard then orders these events according to their proper order of occurrence. When an event is scheduled to occur, the Wizard passes that event back to the originator object, which then determines what its next event should be and when it should occur. For example, a police unit might post an event that places that unit in transit to the scene of an incident. The event specifies the time at which the unit will reach the scene. When the Wizard pulls that event back off the list at the proper time, the originating police unit is notified that it has arrived. The police unit then determines what its next event should be and how long it should take to complete that event. This new information is formed into a new event that is then posted to the event list to be merged with other events from other objects. As a result, the Wizard is continually pulling completed events from the event list and passing them back to the originating object. Those objects then determine what they should do next, format that information into events, and repost these events for the Wizard to find at some later time. All events from all objects are intermixed on the event list, so the Wizard will always find the very next event that should occur, without regard to which object owns the event. No event priority system is applied; all events are ordered only by time of occurrence.

\section{Object Interaction}

Object interactions occur through a combination of object-to-object messages and the custom event list. When one object needs to interact with another, it posts an event that is addressed to the other object. The Wizard pulls that event from the event list and sends it to the receiving object. In most cases, when an object receives a message from another object it will ask the Wizard to remove any events it may have currently posted and to post some new event that is a reaction to the message it received. With the use of this approach, one software object can influence the behavior of another software object. For example, if a police unit is investigating a burglary and the police unit encounters the burglar at the scene, it can say to the burglar, "HALT, you're under arrest!" The burglar will receive the message and, according to its programmed behavior, will either comply or flee. In either case, it will have suspended its search for loot because of the interaction with the police object.

The scenario objects keep the players organized so that the police who are investigating an incident in one part of the city cannot interact with a player in some other location (and scenario).

\section{Range of Incidents}

DISPATCHER contains over 20 different types of possible incidents. The creation of incidents can either be directed from an external text file or be randomly determined by DISPATCHER. Each possible incident type has from five to seven different variations, resulting in more than 120 possible combinations of incidents. The incident descriptions have been extracted from actual emergency dispatch center records, but fictitious names have been substituted for actual ones. The number of unique combinations of incidents that can be provided by DISPATCHER is essentially unlimited. Furthermore, because the incident descriptions are extracted from actual public records, each possible variation represents a completely realistic scenario.

Creating incidents from an external text file provides a means for creating repeatable sequences of incidents. In addition, the text file specifies the type of incident and its scheduled time of occurrence. This provides the experimenter with sequence repeatability as well as control over important parameters such as crime rate and incident types. When the program is permitted to create incidents randomly, it provides a realistic feel for the actual environment encountered by professional dispatchers. Crimes occur at unanticipated times and with widely varying degrees of priority and characteristics.

\section{Scoring}

The dipatcher's performance is scored in two ways. A total of 100 points are possible for each incident that $\mathrm{oc}-$ curs during a session. As an incident progresses, points are subtracted from the initial 100 possible points. The rate of point reduction is a function of the priority of the incident. High-priority incidents lose points faster than do lower priority incidents. The more quickly the dispatcher responds, the more points will remain when that incident is finally resolved. Points for an incident are awarded when the incident is completely resolved. Penalty 
points are subtracted from the point total when various dispatching errors are made. An example of such an error would be the attempt to clear an incident as having been resolved before the police unit had actually resolved it. These error points are generally used to prevent subjects from defeating the scoring system by performing actions that would not be allowed in the real world.

The scoring system has been reviewed by professional dispatchers, who agreed that it is generally an appropriate method.

To maximize a score, the subject wants to complete as many incidents as possible successfully and properly. The more incidents a subject completes, the more points the subject accumulates. DISPATCHER keeps track of both the total number of points earned and the average points per incident.

\section{RESULTS}

DISPATCHER is currently being studied to determine its reliability and validity. Figure 6 shows the first data obtained from DISPATCHER, based on the results of 17

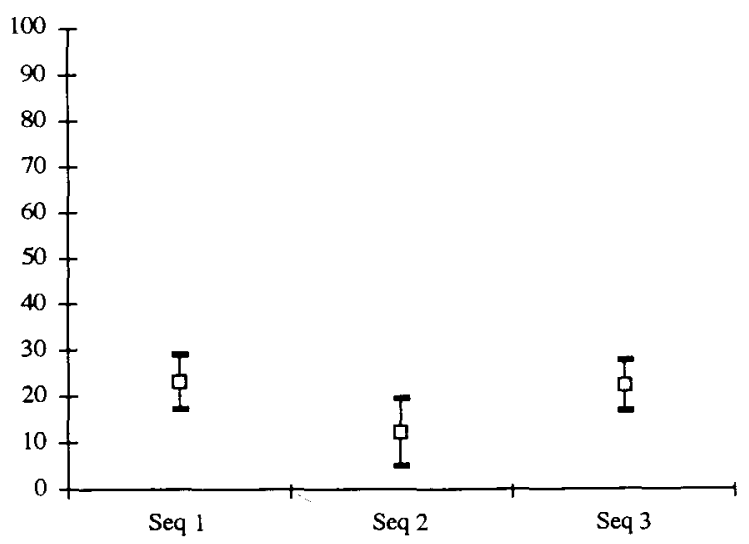

Figure 6. Mean points per incident for three randomly determined sequences.
University of Washington students who were given a 1-h training session and were then tested on DISPATCHER. The data shown are average points per incident achieved for three different sequences of incidents.

The error bars represent one standard error from the mean for each sequence. A comparison of SEQ1 $(M=$ 23.25) with SEQ3 $(M=22.4)$ showed them to be essentially identical $(t=0.20, p=.84)$. While SEQ2 did have a noticeably lower mean $(M=12.4)$, it was not reliably different from SEQ1 $(t=1.62, p=.13)$ or SEQ3 $(t=$ $1.53, p=.15$ ). To place these scores in perspective, scores were obtained from four professional dispatchers. These scores have tended to be in the range of 70-90 points per incident. Direct comparisons of the professional dispatcher scores and the students' scores is not possible, because the data were not collected under exactly the same conditions.

\section{CONCLUSION}

Subjective reviews made by professional dispatchers have been very favorable. On one occasion, after 1-2 min of orientation, an experienced professional dispatcher began using DISPATCHER to teach an inexperienced dispatcher how to handle various specific situations. This apparent comfort with DISPATCHER provides some encouraging face-value validation.

The preliminary results obtained from testing are also encouraging. The scores appear to be reliable. The differences between student subjects and professional dispatchers indicate that situation fidelity and incident realism has been preserved through the process of moving from the field to the laboratory. However, formal judgment of the reliability and validity of DISPATCHER must await the gathering of more extensive data.

\section{REFERENCE}

Webster's New World Dictionary of the American language (2nd ed.) (1976). Cleveland, OH: World Publishing Company. 